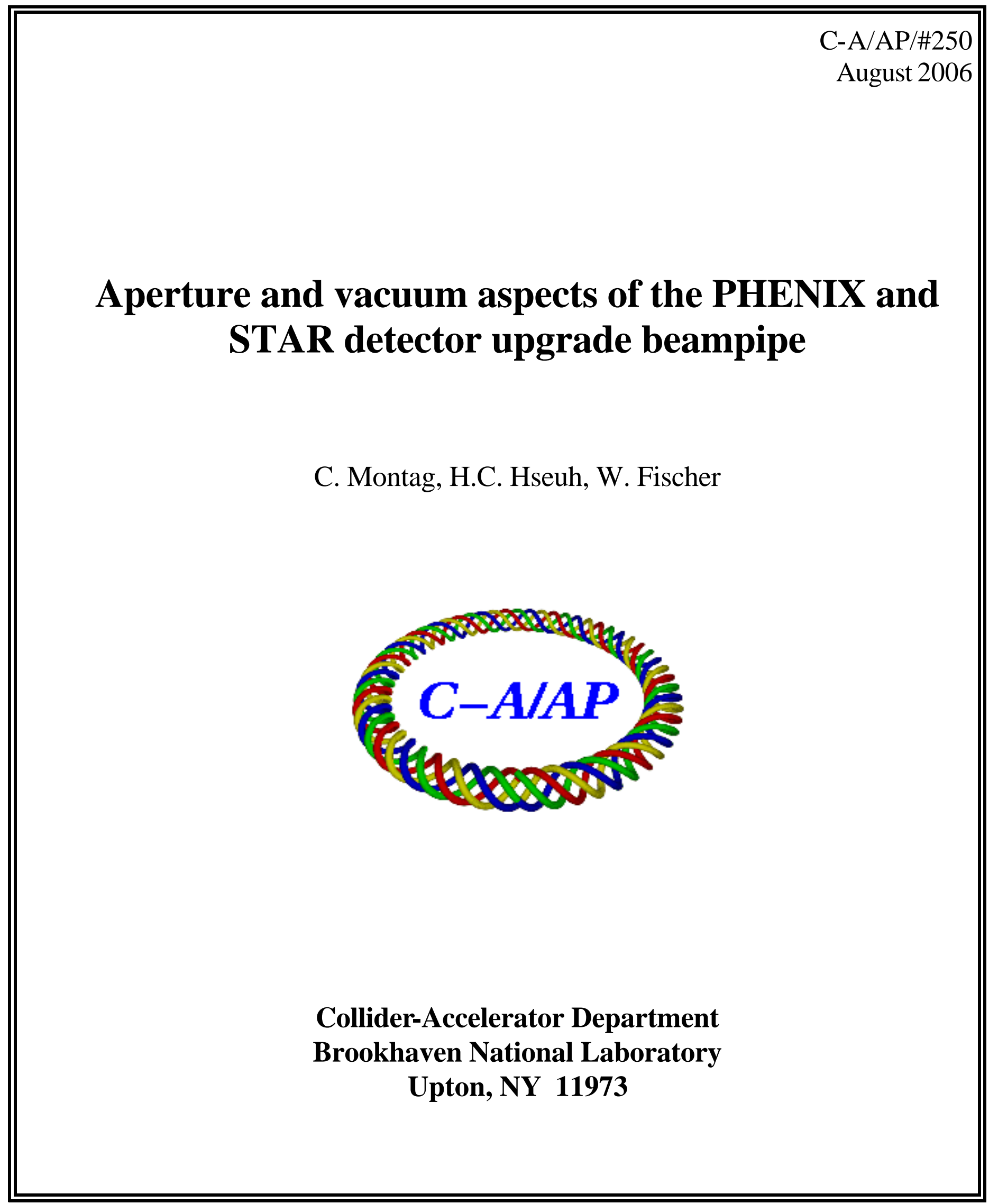


BNL C-A/AP/250

\title{
Aperture and vacuum aspects of the PHENIX and STAR detector upgrade beampipe
}

\author{
C. Montag, H.C. Hseuh, W. Fischer
}

August 17, 2006

\section{Introduction}

As part of the PHENIX and STAR detector upgrade the installation of new beampipes is planned, with an inner radius smaller then the current $38 \mathrm{~mm}$. Based on aperture requirements, we evaluate the minimum inner radius, and estimate the vacuum pressure in the detector region with the reduced radius.

\section{Aperture requirements}

We evaluate the necessary aperture based on two requirements. First, a minimum vertical separation is needed at injection and during the ramp to avoid beam-beam interactions. Second, with circulating beam the detector apertures shall always be in the shadow of another existing element in the ring to provide protection in the event of a large beam loss.

\subsection{Vertical separation requirement}

To avoid beam losses from long-range beam-beam interactions, the beams are vertically separated at injection and during the ramp. Currently, a nominal separation of $\pm 5 \mathrm{~mm}$, not accounting for orbit errors, is maintained in operation. However, even at this separation long-range beam-beam interactions can induce beam losses [1]. Fig. 1 shows the beam loss as a function of vertical separation of two proton bunches at injection, interacting at $s=10.5 \mathrm{~m}$ from the IP. In this case, and with a bunch intensity of $2 \times 10^{11}$ protons in Blue, and $1.8 \times 10^{11}$ protons in Yellow, beam losses are visible when the separation is reduced below $10 \mathrm{~mm}$. Even if no beam loss is observed, the emittance can be affected by long-range beam-beam interactions. To allow for orbit errors, especially at the beginning of the ramp, $\pm 7 \mathrm{~mm}$ vertical separation should be available in all interaction regions.

\subsection{Shadowing requirement}

The straight section between the two triplets is optically a drift without focusing elements, if we neglect the small distortion by the DX separator dipole. Therefore, the beam trajectory in this roughly 50 meters long region is optically equivalent to a straight line. As long as the aperture of the detector beampipe is smaller (in rms beam sizes) than that of the beampipe near the triplets, the detector beampipe is protected from any hits from the stored beam.

Since the interaction point (IP) is an optical symmetry point, $\beta$-functions at a distance $s$ from the IP are

$$
\beta(s)=\beta_{\mathrm{IP}}+\frac{s^{2}}{\beta_{\mathrm{IP}}} .
$$

The largest $\beta$-functions within the detector beampipe therefore occur at its two ends at $s_{d}= \pm 0.4 \mathrm{~m}$, while in the adjacent beampipe they reach their maximum near the triplets, at $s_{t}= \pm 25 \mathrm{~m}$. The beampipe 




Figure 1: Beam losses induced by the long-range beam-beam interaction at injection. The beam loss increases before 10:45 are caused by changes in the tune.

radii at these two locations are $A_{d}=0.02 \mathrm{~m}$ at the detector, and $A_{t}=0.06 \mathrm{~m}$ near the triplet.

Without orbit errors, for the detector beampipe to be in the shadow of the beampipe near the triplets, the relation

$$
\begin{aligned}
& \frac{A_{d}}{\sqrt{\beta_{d}}} \geq \frac{A_{t}}{\sqrt{\beta_{t}}} \\
& \Leftrightarrow \frac{A_{d}}{\sqrt{\beta_{\mathrm{IP}}+\frac{s_{d}^{2}}{\beta_{\mathrm{IP}}}}} \geq \frac{A_{t}}{\sqrt{\beta_{\mathrm{IP}}+\frac{s_{t}^{2}}{\beta_{\mathrm{IP}}}}}
\end{aligned}
$$

needs to be fulfilled. Solving this expression for $\beta_{\mathrm{IP}}$ and inserting the numbers given above yields

$$
\beta_{\mathrm{IP}} \leq 8.8 \mathrm{~m} ;
$$

so the detector beampipe is shielded from the stored beam during stores with $\beta_{\mathrm{IP}}=1.0 \mathrm{~m}$.

Including orbit errors, the worst case scenario occurs when the orbit is parallel shifted by a certain amount $\Delta x$; this amount is the same in the detector beampipe as near the triplets. The free aperture in the plane of the orbit distortion is therefore $A_{d}-\Delta x$ in the detector beampipe, and $A_{t}-\Delta x$ near the triplet. In this case, the detector beampipe is in the shadow of the beampipe near the triplet if the condition

$$
\frac{A_{d}-\Delta x}{\sqrt{\beta_{d}}} \geq \frac{A_{t}-\Delta x}{\sqrt{\beta_{t}}}
$$

is fulfilled. For stores with $\beta_{\mathrm{IP}}=1.0 \mathrm{~m}$, the relevant $\beta$-functions are $\beta_{d}=1.16 \mathrm{~m}$ and $\beta_{t}=626 \mathrm{~m}$, respectively. The condition given in Eq. (5) is fulfilled for

$$
\Delta x \leq 18.2 \mathrm{~mm},
$$

which can be ensure in normal operation.

At injection, however, the condition given in Eq. (4) is not fulfilled. A certain level of protection is provided by aperture limitations elsewhere in the ring, namely the abort kickers in the 10 o'clock IR, 


\begin{tabular}{lcccccc}
\hline \hline device name & $A_{x}$ & $A_{y}$ & $\sqrt{\beta_{x}}$ & $\sqrt{\beta_{y}}$ & $A_{x} / \sqrt{\epsilon \beta_{x}}$ & $A_{y} / \sqrt{\epsilon \beta_{y}}$ \\
& {$[\mathrm{~mm}]$} & {$[\mathrm{mm}]$} & {$[\sqrt{\mathrm{m}}]$} & {$[\sqrt{\mathrm{m}}]$} & {$[\sigma]$} & \\
\hline abort kickers & 25.4 & 38.1 & 6.70 & 12.00 & 6.6 & 5.5 \\
pulsed quadrupole & 32.0 & 12.0 & 3.16 & 3.16 & 17.5 & 6.6 \\
Lambertson & 33.7 & 33.7 & 6.48 & 4.57 & 9.0 & 12.8 \\
new detector beampipes (ID $=40 \mathrm{~mm})$ & 20.0 & 13.0 & 3.16 & 3.16 & 11.0 & 7.1 \\
\hline \hline
\end{tabular}

Table 1: Restricting apertures in RHIC, and $\beta$-functions at injection, with $\beta_{I P}=10 \mathrm{~m}$ in all interaction points. $7 \mathrm{~mm}$ vertical separation bumps have been taken into account at the pulsed quadrupole and the detector beampipe, thus reducing the free aperture by $7 \mathrm{~mm}$. For convenience, the free aperture in $\sigma$ has been calculated assuming a normalized ion beam emittance of $\epsilon_{n}=20 \mathrm{~mm} \cdot \mathrm{mrad}$, and $\gamma=10$ at injection.

the pulsed quadrupole in IP4, and the Lambertson magnets. Tab. 1 lists the apertures of these devices, together with the horizontal and vertical $\beta$-functions at their locations.

Since the free aperture in beam- $\sigma$ for the abort kicker (both planes), the Lambertson (horizontal plane), and the pulsed quadrupole (vertical plane) is smaller than for the proposed detector beampipe, these aperture restrictions provide a certain level of protection during injection. This protection is lost if the detector beampipe becomes the limiting aperture in the machine.

Abort kicker misfires at store lead to single-turn losses. The location of those losses around the perimeter of the ring is determined by the betatron phase advance between kicker and loss area, as well as the $\beta$-function and physical aperture at the location of the loss. In RHIC, the abort kickers deflect the beam on the downstream side of IR10; therefore, there is at least one entire arc between these kickers and the PHENIX or STAR detector. Within that arc, there is at least one location where the $\beta$-function reaches its maximum of approximately $50 \mathrm{~m}$, while the betatron phase advance between the kickers and this location is close to $n \cdot \pi+\pi / 2$. With the beampipe inner radius in the arcs being $35 \mathrm{~mm}$, a particle deflected by a mis-firing abort kicker would hit this area in the arc first, instead of hitting the $20 \mathrm{~mm}$ ID detector beampipe, where the $\beta$-function is $1.0 \mathrm{~m}$. The aperture ratio in this case is $\sqrt{50 / 1.0} \cdot 20 / 35=4.0$.

With a $20 \mathrm{~mm}$ inner radius beampipe in PHENIX and STAR, the same aperture is available as in IP4 now. If a smaller beampipe would be installed in either experiment, these could become the limiting aperture at injection. kicker.

\section{Vacuum considerations}

The changes in vacuum pressure inside the new detector beam pipes can be analyzed numerically and compared with that of the present beam pipes. We assume the following conditions for the analysis: First, the desorption rate along the pipe is uniform and independent of the radius; this is a reasonable assumption based on uniform electron density which generates the observed dynamic pressure rise. Second, the desorbed gas flows unidirectionally toward the pumps located $7.5 \mathrm{~m}$ from interaction points, such that the pressure profile becomes symmetric around the IP. The beam pipes closer to the ion pumps have a radius of $6.1 \mathrm{~cm}$, transitioned to $3.6 \mathrm{~cm}$ towards the IP. The pressure distribution is slightly worse at the 8 o'clock side due to the longer $3.6 \mathrm{~cm}$ radius section. We will use the 8 o'clock side in the analysis, which has $4.0 \mathrm{~m}$ long $6.1 \mathrm{~cm}$ radius pipes and $3.5 \mathrm{~m}$ long $3.6 \mathrm{~cm}$ radius pipes. In the proposed upgrade, the $3.6 \mathrm{~cm}$ radius pipe will be shortened to $3.0 \mathrm{~m}$, with the $0.5 \mathrm{~m}$ long section at the IP replaced by a $2.0 \mathrm{~cm}$ radius pipe.

The pressure at any location depends on the linear conductance of the beam pipes and the distance 
Table 2: Linear conductance $C o$ for present and planned PHENIX and STAR beam pipe configurations.

\begin{tabular}{lcccc}
\hline \hline quantity & unit & \multicolumn{3}{c}{ values } \\
\hline $\mathrm{R}$ & $\mathrm{cm}$ & 6.1 & 3.6 & 2.0 \\
$\mathrm{Co}\left(\mathrm{H}_{2}\right)$ & $1 \cdot \mathrm{cm} / \mathrm{s}$ & $8.4 \cdot 10^{4}$ & $1.7 \cdot 10^{4}$ & $3 \cdot 10^{3}$ \\
$\mathrm{Co}(\mathrm{CO})$ & $1 \cdot \mathrm{cm} / \mathrm{s}$ & $2.2 \cdot 10^{4}$ & $4.6 \cdot 10^{3}$ & $8 \cdot 10^{2}$ \\
\hline \hline
\end{tabular}

Table 3: Effective pumping speed at IP.

\begin{tabular}{lcc}
\hline \hline & $\mathrm{S}\left(\mathrm{H}_{2}\right)[\mathrm{l} / \mathrm{sec}]$ & $\mathrm{S}(\mathrm{CO})[\mathrm{l} / \mathrm{sec}]$ \\
\hline Now & 36 & 10 \\
Upgrade & 24 & 6.6 \\
\hline \hline
\end{tabular}

from the pump. The linear conductance $C o($ in $1 \cdot \mathrm{cm} / \mathrm{s})$ is given by

$$
C o=30.5 \cdot R^{3}(T / M)^{0.5}
$$

where $R$ is the beam pipe radius (in $\mathrm{cm}$ ), $T$ the ambient temperature (in $\mathrm{K}$ ), and $M$ molecular weight of the residual gas. In Tab. 2 the linear conductance $C o$ is given for $\mathrm{H}_{2}$ and $\mathrm{CO}$, and various beam pipe radii $R$.

The effective pumping speed $S$ (in l/s) from the IP toward the pump, which has an estimated pumping speed of $250 \mathrm{l} / \mathrm{sec}$ for $\mathrm{CO}$ and $400 \mathrm{l} / \mathrm{sec}$ for $\mathrm{H}_{2}$, respectively, can be obtained by dividing $C o$ by the corresponding section length $L$, and combining them together, similar to the total resistance of resistors in series. Table 3 lists the resulting total pumping speeds for the present as well as the proposed upgrade configuration, for both $\mathrm{H}_{2}$ and $\mathrm{CO}$.

This simplified approach gives a 33\% reduction in effective pumping speed, and, correspondingly, a $50 \%$ increase in pressure at IP, when the beam pipe radius is reduced from its current value to $R=20 \mathrm{~mm}$.

This increase may be significant, however, the new PHENIX and STAR central pipes will be NEG coated, resulting in a lower secondary electron yield and a lower electron stimulated desorption coefficients, and therefore a lower dynamic pressure rise.

\section{Conclusions}

The aperture requirements of new detector beam pipes were evaluated based on the need for a sufficiently large vertical separation to avoid long-range beam-beam interactions, and the requirement that the detector beam pipe aperture is always in the shadow of another existing aperture in the ring. Based on these criteria we recommend not to consider detector beam pipes with an inner radius of less than $20 \mathrm{~mm}$.

With the reduced radius an increase in the vacuum pressure in the detector beam pipe of $50 \%$ is expected if no further vacuum improvements are made. The new beam pipes should be NEG coated, and NEG activation at $200^{\circ} \mathrm{C}$ should be possible. With these improvement we expect the vacuum to be no worse than under the current conditions.

\section{References}

[1] W. Fischer, R. Alforque, H.C. Hseuh, B. Lambiase, C.J. Liaw, G. Miglionico, T. Russo, J.-P. Koutchouk, F. Zimmermann, T. Sen, "Measurement of the long-range beam-beam effect at injection, and design for a compensator in RHIC", BNL C-A/AP/236 (2006). 